\title{
MRI DIAGNOSIS OF TWO CASES OF FIBROLIPOMATOUS HAMARTOMA OF THE MEDIAN NERVE WITH HISTOPATHOLOGICAL CORRELATION
}

\author{
Mary Hazarika Bhuyan¹, R. K. Bhuyan², Mondita Borgohain³, Mrinal Baruah
}

\section{HOW TO CITE THIS ARTICLE:}

Mary Hazarika Bhuyan, R. K. Bhuyan, Mondita Borgohain, Mrinal Baruah. "MRI Diagnosis of Two Cases of Fibrolipomatous Hamartoma of the Median Nerve with Histopathological Correlation". Journal of Evolution of Medical and Dental Sciences 2015; Vol. 4, Issue 64, August 10; Page: 11275-11280,

DOI: $10.14260 /$ jemds/2015/1627

ABSTRACT: Fibrolipomatous hamartoma is an uncommon congenital disorder, which is characterized by disproportionate hyperplasia of adipose tissue infiltrating along the perineurium, the epineurium and the affected nerve trajectory. ${ }^{1}$ The median nerve and its branches are most commonly affected, followed by the radial nerve, ulnar nerve, nerves at the dorsal aspect of the foot, brachial plexus and cranial nerves. ${ }^{1,2}$ Magnetic resonance imaging demonstrated a typical fibrolipomatous hamartoma with high signal intensity of fat on both T1-weighted and T2-weighted images, characteristic coaxial cable appearance on axial images, and spaghetti appearance on sagittal images in two of our cases. Fibrolipomatous hamartoma (FLH) of nerve is a rare tumor-like condition in which mature fat infiltrates the neural sheath, with the majority of the lesions occurring in the median nerve. ${ }^{3} \mathrm{We}$ present two cases of Fibrolipomatous hamartoma of median nerve at the wrist with an unusual proximal extension to the forearm in one case.

KEYWORDS: Fibrolipomatous hamartoma, MRI.

INTRODUCTION: Fibrolipomatous hamartoma which is a rare benign tumor, characterized by an anomalous overgrowth of fibroadipose tissue between and around nerve bundles, which causes enlargement of the affected nerve and form a palpable neurogenic mass. ${ }^{2}$ (WHO) grouped intraneural lipoma, fibrolipomatous hamartoma of the nerve, fatty infiltration, and neural fibrolipoma under Lipomatosis of the Nerve_in 2002.

\section{CASE REPORTS:}

CASE 1: A 21 year old man was referred to our department of radiodiagnosis from general surgery. He had a swelling in the palmar aspect of right hand (Fig. 1A, B) in the thenar aspect with mild pain. It was a palpable, non-tender mass extending to the forearm (Fig. 1B yellow arrow). The slowly growing mass had been noticed since his birth, which was gradually increasing in size, presently with pain. It was associated with recent onset of paresthesia of radial three fingers. Sensory deficit was noted in the right middle finger in addition to disorderedly thumb opposition Clinical diagnosis was lipoma. However for the oblong extension (Fig. 1B yellow arrow) to the forearm subjected the man for an MRI study. Plain $\mathrm{X}$ ray of the hand did not show any evidence of periosteal or endosteal new bone formation (Fig. 2).

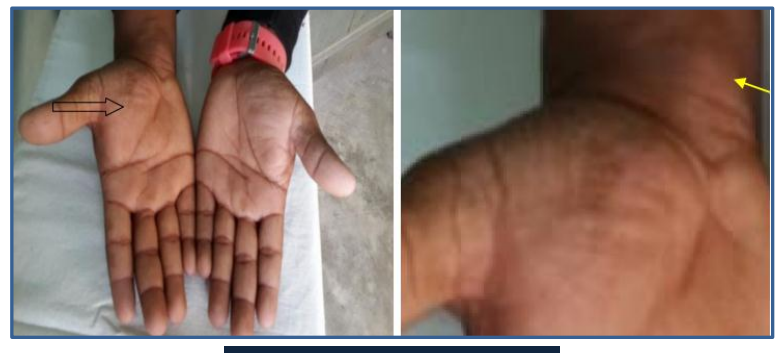

Figure $1 \mathrm{~A} \& 1 \mathrm{~B}$ 


\section{CASE REPORT}

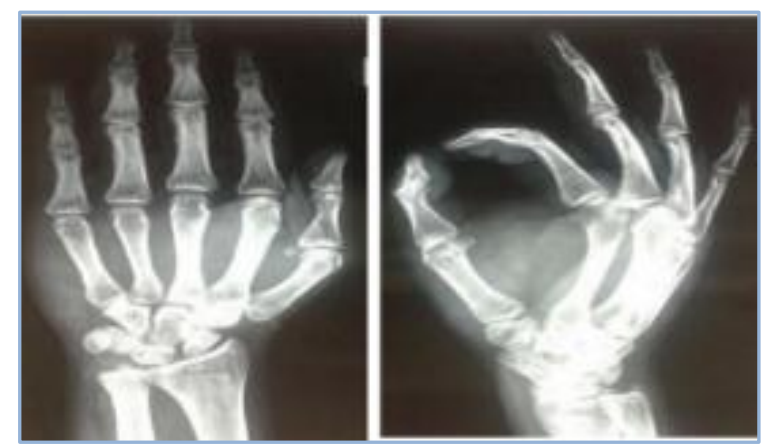

Figure 2

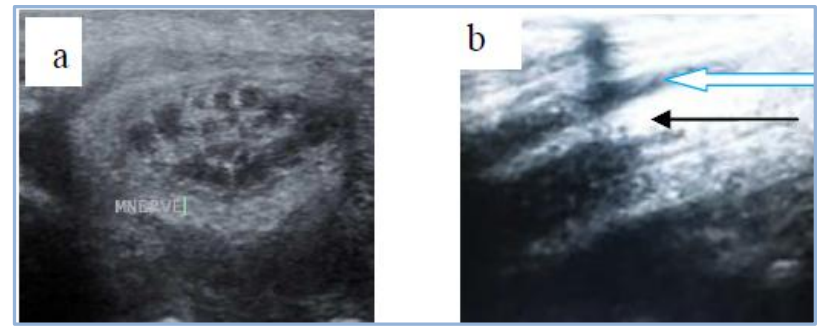

Figure 3A \& 3B

Radiographs of the affected hand, wrist and forearm revealed prominent low density soft tissue surrounding the $1^{\text {st }}$ and $2^{\text {nd }}$ metacarpal, which extended to the volar aspect of the right wrist (Fig. 2). Ultrasonography of right median nerve shows hypoechoic thickened axonal bundles (Fig. 3B, blue arrow) interspersed in echogenic fatty tissue (Fig. 3A, black arrow) giving "co-axial cable" like appearance Sonography shows the characteristic hypoechoic coaxial cabling encased by an echogenic substratum. ${ }^{4}$

MRI showed a mass lesion in the thenar eminence which was suppressed on STIR images (Fig. $4 \mathrm{~A}, \mathrm{C}$ ). The mass is seen infiltrating into nearby flexor retinaculum and medial fibres of the thenar group of muscles. The fatty tissue extends along the median nerve into the index and middle finger.

Superiorly the fatty tissue deposition is extending along the radial aspect of forearm along hypertrophic median nerve (Fig. 4C). Post contrast study with gadolinium show few enhancing areas in the median nerve along with normal muscular enhancement (Fig. 4D, arrows).

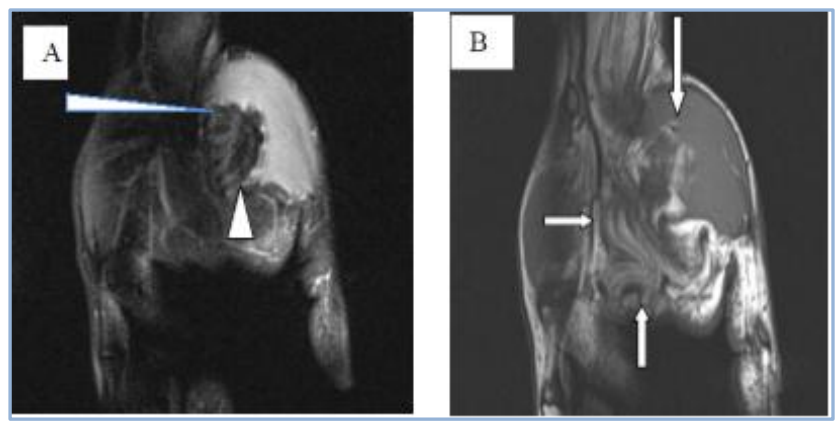

Figure 4A \& 4B 


\section{CASE REPORT}

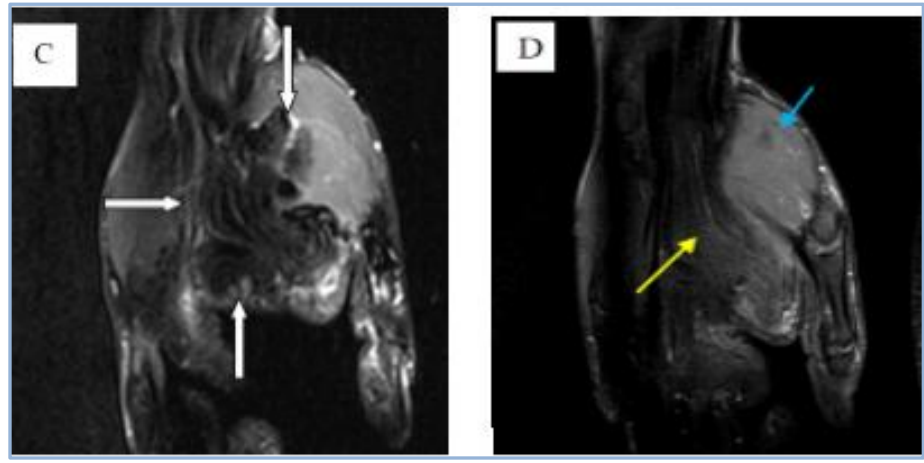

Figure 4C \& 4D

The mass lesion in between arrowheads shows typical fat suppression in coronal proton density fat supressed spin echo images (A), in T2Wspin echo fat suppressed coronal image (C), hyperintense in T2W spin echo coronal image (B), with mild enhancement of the intervening septae (yellow arrow), normal muscular enhancement (blue arrow) in T1W fat suppressed spin echo images(D).

The MRI features were consistent with a thenar lipoma with infiltration into the median nerve conforming to median nerve lipomatosis/Fibrolipomatous Hamartoma.
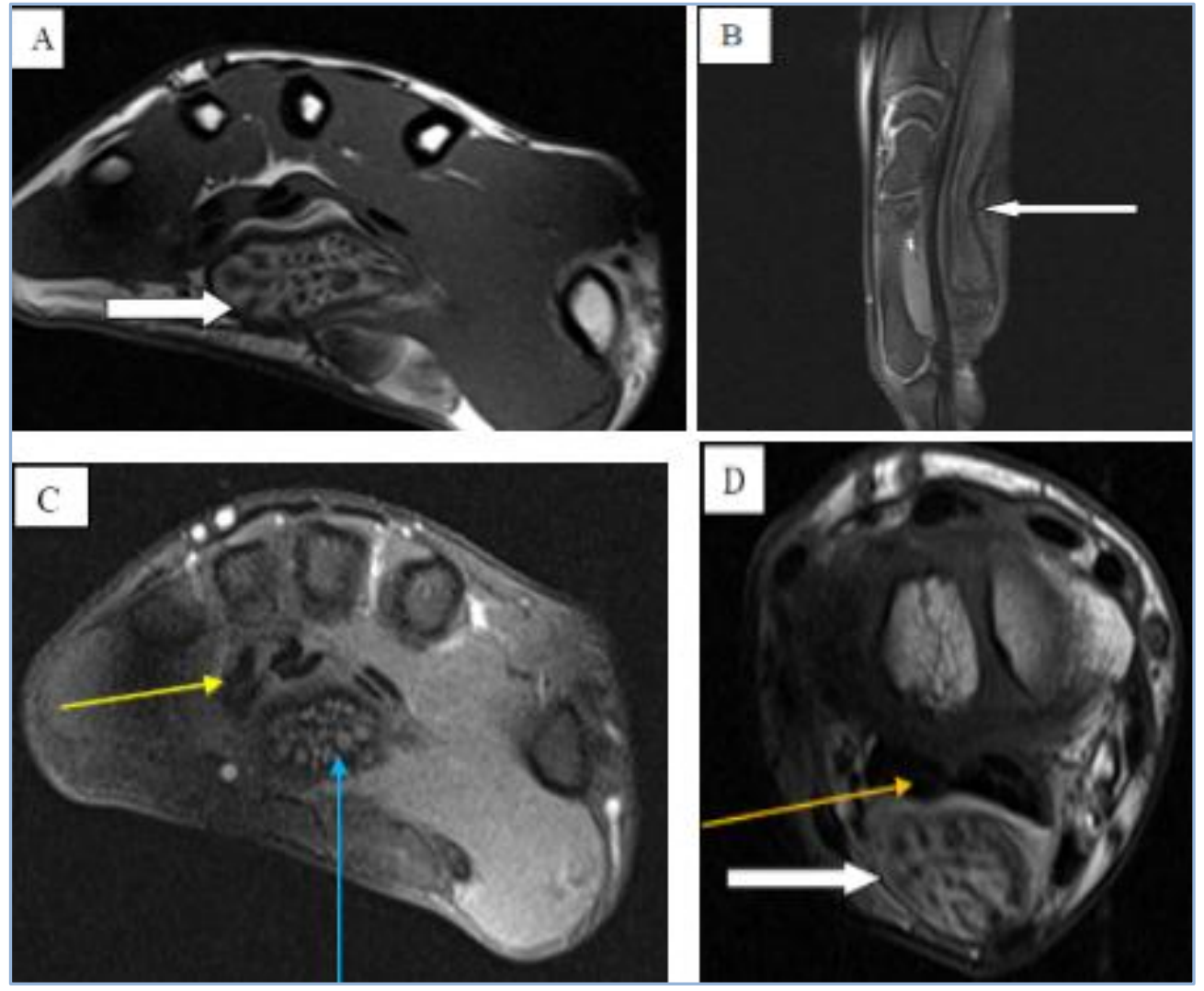

Figure 5A, 5B, 5C \& 5D 


\section{CASE REPORT}

Fibrolipomatous hamartoma of the median nerve, Axial spin echo T1-weighted imaging of the: (A) wrist and (D) distal forearm shows the enlarged median nerve with characteristic coaxial cable appearance (White arrows in axial images A, D and sagittal image B) of fibrolipomatous hamartoma and dorsal displacement of the flexor tendons (yellow arrows in C, D). Axial fat-suppressed gadolinium enhanced spin echo T1-weighted imaging of the wrist show fat suppression and poor contrast enhancement (C, Blue arrow) On sagittal scan give spaghetti-like appearance of enlarged median nerve (white arrowB)

CASE 2: Another 10 year old boy was referred from the department of pediatric surgery. There was a swelling in the thenar eminence of left hand (Fig. 6a). There was no discoloration, nevus, skin defect, ulceration or pitting edema over the mass. Thenar swelling was soft and mildly tender on palpation with no thrill. There was no disturbance of sensory or motor functions. No evidence of any focal enlargement of digits noted.

The clinical diagnosis was haemangioma. Plain Radiograph shows mild soft tissue swelling with normal underlying bones (Fig. 6b). On Axial and Longitudinal HR USG scan shows hypoechoic thickened axonal bundles interspersed in echogenic fatty tissue (Yellow arrow heads). (Fig. 6c \& d)

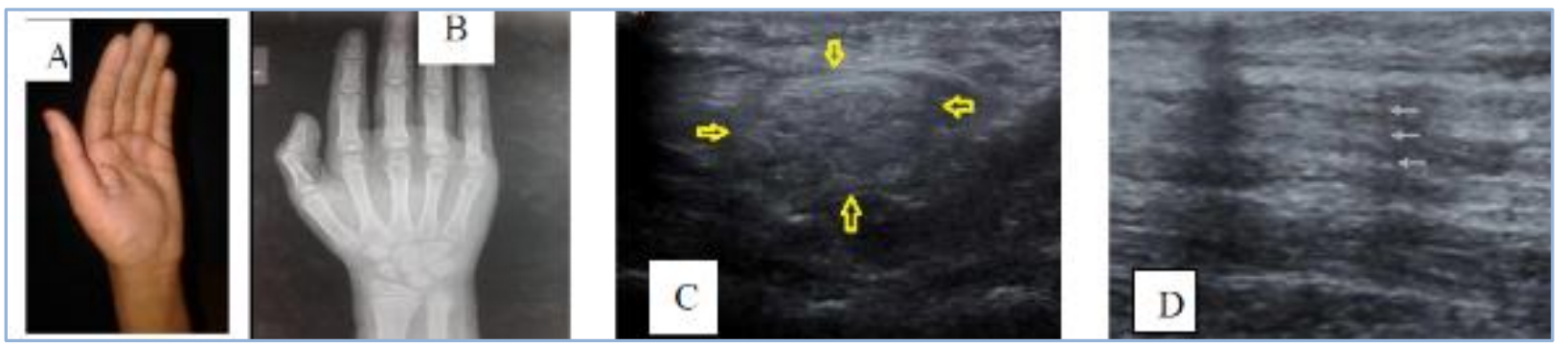

Figure 6A, 6B, 6C \& 6D

Figure 6C, 6D Axial and Longitudinal HR USG scan hypoechoic thickened axonal bundles interspersed in echogenic fatty tissue (Yellow arrow heads).
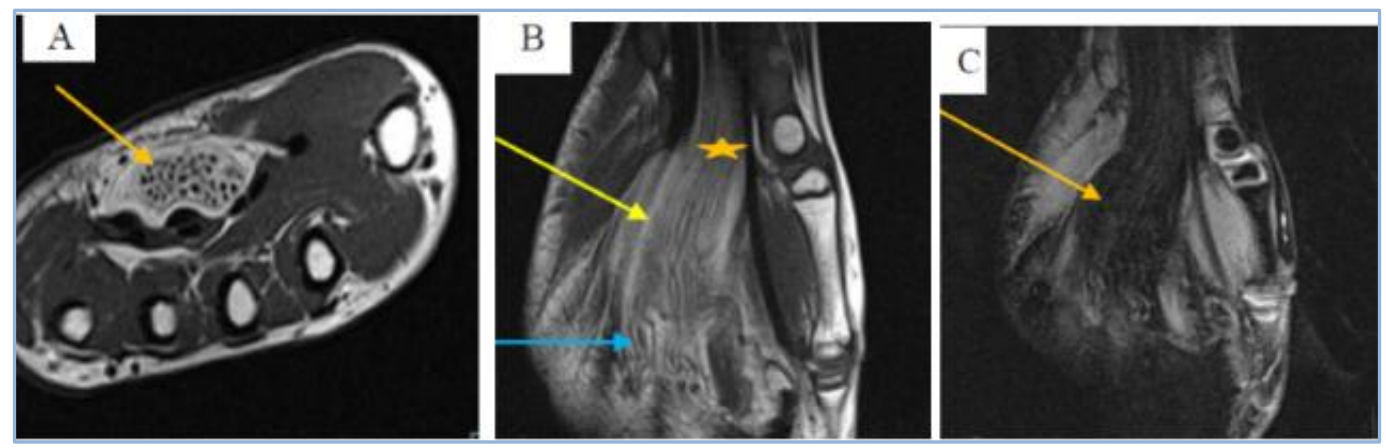

Figure 7A, 7B \& 7C

Figure 7 Median nerve lipomatosis, T1weighted axial image (A) and coronal image (B), shows enlarged median nerve in the palm (Yellow arrow) and left wrist (Yellow star) which is suppressed on STIR sequences seen in Proton density spin echo coronal image(C). 
On MRI, TI hyperintense mass noted along the course of the left meadin nerve (Fig. 7A, B). The area is suppressed on STIR (Short tau inversion recovery) sequences (Fig. 7C) which indicates the presence of fat signals. The fatty deposition is extending into the respective nerve tributaries of $2^{\text {nd }}$ and $3^{\text {rd }}$ digits (Fig. 7B blue arrow). There is a fusiform mass (Yellow star Fig. 7B) in the expected location of the median nerve that is continuous with the nerve proximally and distally. On the nonfatsuppressed images the high signal represents the fatty tissue infiltrating and enlarging the median nerve which drops completely with fat suppression (Fig. 7C) with no abnormal post contrast enhancement (Fig. 8A).
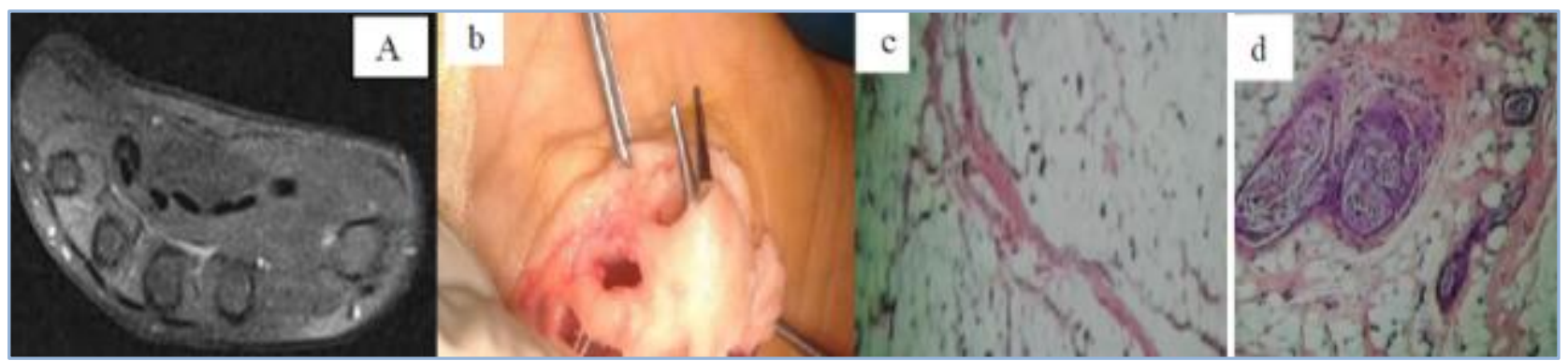

\section{Figure 8A, 8B, 8C \& 8D}

Figure 8. Histopathological samples confirming lipomatous tissue surrounding the nerve fascicles (C, D) Intraoperative surgical specimen (B) enlarged fusiform median nerve with fibrofatty tissue proliferation extended from the distal forearm to mid palm.

DISCUSSION: Fibrolipomatous hamartomas are benign tumors usually affecting commonly children and young adults, the development of FLH includes the proliferation of mature fatty and fibrous tissues within the epineurium and perineurium of a major nerve. ${ }^{2}$

The most common presentation is that of a soft, mobile, slowly enlarging and often asymptomatic mass on the volar wrist or forearm that follows the distribution of a nerve often present since infancy. Occasionally compression neuropathy with the primary complaint of severe pain, paresthesia and thenar motor weakness is present.

Sonographic findings show smooth, rounded, thickened hypoechoic or anechoic fascicles surrounded by echogenic fatty tissue corresponding to histological findings. No intralesional flow is seen on color Doppler.

MRI Features of FLHs including the coaxial cable-like appearance on axial sections, and a spaghetti-like appearance on coronal images. Serpentine-like appearance of fibroadipose tissue intermingled with nerve fascicles. Transverse sections show thin hypointense septa within the fat tissue that separate some nerve bundles, what we refer to as the "lotus sign."

These septa may represent thickened perineurium, a characteristic pathological finding. These differences in MR imaging would be useful for the differential diagnosis between intraneural lipoma and fibrolipomatous hamartoma of the nerve, because even the biopsy data might be sometimes not informative. ${ }^{5}$ Gold standard treatment-conservative approaches including decompression and debulking of the fibrofatty sheath, microsurgical dissection of the neural elements and observation for asymptomatic patients. ${ }^{5}$ 


\section{REFERENCES:}

1. Nouira K, Belhiba H, Baccar S, Miaaoui A, Ben Messaoud M, Turki I, Cheour I, Menif E. Fibrolipoma of the median nerve. Joint Bone Spine. 2007; 74(1): 98-9.

2. So CK, Tam KF, Lui CY, Lee CM. Fibrolipomatous hamartoma of the Median Nerve. J HK Coll Radiol. 2007; 10: 81-84.

3. V. Singla, V Virmani, P Tuli, ${ }^{*}$ P Singh, and N Khandelwal Indian J Radiol Imaging. 2008 Nov; 18(4): 298-301. doi: 10.4103/0971-3026.43844.

4. Toms AP, Anastakis D, Bleakney RR, Marshal TJ. Lipofibromatous hamartoma of the upper extremity: a review of radiologic findings for 15 patients. AJR Am J Roentgenol. 2006; 1 86: 805811.

5. Taketo Okubo,a,b,* Tsuyoshi Saito, ${ }^{\mathrm{b}}$ Hiroyuki Mitomi, ${ }^{\mathrm{b}}$ Tatsuya Takagi, ${ }^{\mathrm{a}}$ Tomoaki Torigoe, Int J Surg Case Rep. 2012; 3(9): 407-411.Published online 2012 May 23. Doi: 10.1016/j.ijscr.2012.05.007.

\section{AUTHORS:}

1. Mary Hazarika Bhuyan

2. R. K. Bhuyan

3. Mondita Borgohain

4. Mrinal Baruah

\section{PARTICULARS OF CONTRIBUTORS:}

1. Associate Professor, Department of Radio-diagnosis, Assam Medical College and Hospital, Dibrugarh.

2. Professor, Department of General Surgery, Assam Medical College and Hospital, Dibrugarh.

FINANCIAL OR OTHER COMPETING INTERESTS: None
3. Professor, Department of Pathology, Assam Medical College and Hospital, Dibrugarh.

4. Pathologist,

\section{NAME ADDRESS EMAIL ID OF THE} CORRESPONDING AUTHOR:

Dr. Mary Hazarika Bhuvan,

PQ 12. Lane I,

Assam Medical College,

Dibrugarh-786002, Assam, India.

E-mail: maryhazarikabhuyan@yahoo.com

Date of Submission: 16/07/2015.

Date of Peer Review: 17/07/2015.

Date of Acceptance: 04/08/2015.

Date of Publishing: 10/08/2015. 